Fisioter Bras 2018;19(1):19-27

\title{
ARTIGO ORIGINAL \\ Comparação da função ventilatória e da atividade elétrica dos músculos inspiratórios acessórios entre adultos jovens com respiração nasal e oral \\ Comparison of ventilatory function and electrical activity of the accessory inspiratory muscles in young adults with nasal and mouth breathing
}

Maria Elaine Trevisan, D.Sc. ${ }^{*}$ Jalusa Boufleur, M.Sc. ${ }^{* *}$, Juliana Corrêa Soares, M.Sc. ${ }^{* *}$, Rodrigo Agne Ritzel ${ }^{* * *}$, Ana Maria Toniolo da Silva, D.Sc. ${ }^{* * * *}$, Eliane Castilhos Rodrigues Corrêa, D.Sc.*

${ }^{*}$ Profa. do Departamento de Fisioterapia e Reabilitação(UFSM), Santa Maria/RS, ${ }^{*} U F S M$, Santa Maria/RS, ${ }^{* * *} F a c u l d a d e$ Metodista de Santa Maria, Santa Maria/RS, ${ }^{* * * * H o s p i t a l}$ Universitário de Santa Maria (UFSM), Santa Maria/RS, ${ }^{* * * *}$ Prof. Departamento de Fonoaudiologia UFSM

Recebido em 29 de novembro de 2016; aceito em 30 de novembro de 2017.

Endereço para correspondência: Maria Elaine Trevisan, Rua Silva Jardim 2141/701, 97010493 Santa Maria RS, E-mail: elaine.trevisan@yahoo.com.br, Jalusa Boufleur: jalusaboufleur@yahoo.com.br, Juliana Correa Soares: jjuzinha83@yahoo.com.br; Rodrigo Agne Ritzel: rodrigo.ritzel@uol.com.br; Ana Maria Toniolo da Silva: amariatoniolo@gmail.com; Eliane Castilhos Rodrigues Corrêa: eliftrs@yahoo.com.br

\section{Resumo}

Objetivo: Avaliar a função ventilatória, o pico de fluxo inspiratório nasal, as pressões respiratórias máximas, a atividade elétrica dos músculos inspiratórios acessórios e a relação entre as variáveis, em adultos com respiração oral (RO), comparando-os com adultos com respiração nasal (RN). Métodos: Estudo transversal, controlado com 77 participantes, entre 18 e 30 anos, ambos os sexos, distribuídos em Grupo RO $(n=38)$ e $R N(n=39)$. Foram avaliados por espirometria, manovacuometria, para obtenção da Pressão Inspiratória Máxima (PImáx) e Pressão Expiratória Máxima (PEmáx); medida do pico de fluxo inspiratório nasal (PFIN) e eletromiografia de superfície (EMG) dos músculos esternocleidomastoideo (ECM) e trapézio superior durante testes de PImáx e PFIN. Resultados: O grupo RO apresentou valores significativamente menores de PImáx (91 versus 100\%), PEmáx (91,6 versus 102,6\%) e PFIN $(121,4$ versus $147,7 \mathrm{I} / \mathrm{min})$. Houve diferença $(p<0,01)$ na atividade EMG do $E C M$, com valores menores no grupo RO. A PImáx se correlacionou com o PFIN $(r=0,48, p<0,01)$. Conclusão: Os RO apresentaram menores valores de PFIN, PImáx, PEmáx e de amplitude da atividade EMG dos músculos inspiratórios acessórios durante inspirações rápidas, quando comparados aos RN. Traz como contribuição para a prática clínica, a importância de uma investigação global do modo respiratório, não envolvendo apenas intervenções médicas, ortodônticas e miofuncionais, mas também os aspectos ventilatórios e musculares, sobre os quais se identificou a influência da RO.

Palavras-chave: músculos respiratórios, espirometria, eletromiografia, obstrução nasal, respiração bucal.

\footnotetext{
Abstract

Objective: To evaluate the ventilatory function, peak nasal inspiratory flow, respiratory muscle strength, electrical activity of the accessory inspiratory muscles and the relationship between the variables comparing mouth (MB) and nasal-breathing (NB) adults. Methods: Consisted in a cross-sectional, controlled study with 77 participants, between 18 and 30 years old, both sexes, distributed into MB $(n=38)$ and NB $(n=39)$ group. They were assessed by manovacuometry, for acquisition of the Maximal Inspiratory Pressure (MIP) and Maximal Expiratory Pressure (MEP) measures; peak nasal inspiratory flow meter (PNIF) and, surface electromyography (sEMG) of the of the sternocleidomastoid (ECM) and upper trapezius muscles, during MIP and PNIF. Results: The MB group presented values significantly lower on the MIP (91 versus $100 \%$ ), MEP (91.6 versus $102.6 \%)$ and PNIF (121.4 versus $147.7 \mathrm{l} / \mathrm{min})$. There was difference $(p<0.01)$ in the ECM muscle activity, with lower values in the MB group. The MIP measure correlated to the PNIF ( $r=0.48, p<0.01)$. Conclusion: The adults MB presented smaller values of PNIF, MIP, MEP,
} 
and EMG amplitude of accessory inspiratory muscles during fast inspiration when compared to the NB. As a contribution to clinical practice, the importance of a global investigation of the respiratory mode, involving not only medical, orthodontic and myofunctional interventions, but also the ventilatory and muscular aspects, on which the influence of RO was identified.

Key-words: respiratory muscles, spirometry, electromyography, nasal obstruction, mouth breathing.

Introdução

A respiração nasal $(\mathrm{RN})$ é inata ao ser humano e, ao passar pelas fossas nasais, o ar é preparado para atingir estruturas mais nobres do sistema respiratório. Além disso, é essencial ao crescimento, desenvolvimento e funcionalidade dos sistemas craniocervical e estomatognático [1].

Qualquer fator que dificulte a permeabilidade nasofaríngea faz com que a RN seja, parcialmente ou totalmente, suplementada pela respiração oral (RO). Esses fatores podem ser obstrutivos, especialmente pela hipertrofia dos tecidos adenotonsilares, ou funcionais, decorrentes de edema transitório da mucosa nasal, flacidez muscular ou manutenção do hábito oral, mesmo após correção cirúrgica da obstrução nasal [2]. A suplência oral é uma adaptação anormal e ineficiente da RN induzindo a desequilíbrios funcionais, posturais, biomecânicos e oclusais [3].

Assim, a obstrução do espaço aéreo nasofaríngeo esta associada à postura anteriorizada da cabeça, para facilitar a passagem de ar da nasofaringe ao trato respiratório inferior. Essa adaptação, que resulta em desequilíbrio muscular e alteração no eixo postural, é frequentemente encontrada em respiradores orais $(R O)[1,3,4]$, refletindo na cinética do diafragma $[1,3,4]$ e da caixa torácica [3,5]. Adicionalmente, estudos envolvendo $R O$, encontraram maior atividade da musculatura respiratória acessória no repouso [6,7], fraqueza muscular respiratória [3,5,8,9], hipoatividade dos músculos abdominais, predominância do movimento inspiratório no tórax superior [4], redução da Capacidade Vital Forçada (CVF) [10,11], capacidade de exercício e qualidade de vida [8].

O diagnóstico da obstrução nasal é fundamentado na história clínica da doença nasal, no exame físico e, em alguns casos, complementado com a endoscopia nasossinusal [12,13]. Outro recurso de avaliação complementar é a medida do pico de fluxo inspiratório nasal (PFIN), um método não invasivo, de fácil manuseio e baixo custo, que mede de forma indireta a obstrução nasal, considerando que o aumento da resistência nasal modifica o fluxo aéreo nasal e, consequentemente, seu pico [14].

A espirometria é frequentemente utilizada para a avaliação dos volumes e capacidades pulmonares em indivíduos com sintomas respiratórios ou doença respiratória conhecida. Os parâmetros espirométricos correspondem a uma manobra expiratória forçada a partir da Capacidade Pulmonar Total (CPT) [15].

A força gerada pela contração dos músculos respiratórios, frequentemente, é mensurada por meio das pressões respiratórias máximas. A monitorização da pressão inspiratória máxima (PImáx) e expiratória máxima (PEmáx) possui relevância para a prática clínica, pois pode confirmar a disfunção dos músculos respiratórios em diversas situações [1618]. Outra possibilidade de avaliar a função muscular é pela eletromiografia de superfície (sEMG), sendo um complemento diagnóstico na avaliação funcional do sistema muscular respiratório [19]. Representa o sinal elétrico gerado pelos músculos esqueléticos, detectado na superfície da pele. Tem uma utilização relativamente simples e ampla aceitação pelo fato do eletrodo de superfície ser minimamente invasivo.

O objetivo do estudo foi avaliar a função ventilatória, o pico de fluxo inspiratório nasal, as pressões respiratórias máximas, a atividade elétrica dos músculos inspiratórios acessórios e a relação entre as variáveis em adultos com respiração oral, comparando-os com respiradores nasais.

A hipótese do estudo é de que exista diferença conforme o modo ventilatório, ou seja, respiradores orais apresentam menores valores no PFIN, nas pressões respiratórias máximas e nas variáveis espirométricas e maior recrutamento da musculatura acessória da respiração, em relação aos respiradores nasais. 
Estudo exploratório, transversal, controlado, realizado na Universidade Federal de Santa Maria, Santa Maria/RS, aprovado pelo Comitê de Ética local (nº 04039912700005346) e Termo de Consentimento Livre e Esclarecido assinado por todos os indivíduos.

A amostra foi selecionada de forma não probabilística e intencional. Participaram adultos de ambos os sexos, idade entre 18 e 30 anos, com respiração oral (grupo RO) que foram comparados com um grupo de controle com respiração nasal (grupo RN), índice de massa corporal (IMC) entre 18,5 e 24,9 kg/m2, sem evidência de doença pulmonar, neuromuscular e/ou deformidades torácicas, não tabagistas e/ou expostos a ambiente de risco. Foram excluídos indivíduos em uso de corticosteróides, relaxantes musculares, vasoconstritores nasais, gripe ou crise alérgica no dia da avaliação, cirurgia torácica, abdominal, nasossinusais e hérnia abdominal.

- Diagnóstico do modo respiratório: baseado na anamnese, sinais e sintomas, características físicas relacionados com a RO, como ausência de selamento labial, lábios hipotônicos, face alongada, manutenção da boca aberta a maior parte do dia e/ou sono, sialorreia noturna e ronco [4,8]. A avaliação otorrinolaringológica [12,13] incluiu a nasofibroscopia com avaliação da presença de obstrução das vias aéreas superiores por hipertrofia das tonsilas palatinas e/ou faríngeas ou rinite.

- Massa corporal e estatura: a massa corporal (balança digital, marca Camry, modelo EB9013) foi aferida com o indivíduo em pé, postura estática, descalço, vestindo o mínimo de roupa, braços ao longo do corpo, cabeça e olhos voltados para frente. Para medir a estatura empregou-se um estadiômetro de madeira com resolução de 0,1 mm, estando os indivíduos na posição em pé, eretos, descalços, olhar na horizontal, calcanhares, nádegas e coluna dorsal em contato com o aparelho. Foi solicitada uma inspiração profunda sustentada por alguns segundos, sendo o cursor colocado no vértice da cabeça e feita a leitura da estatura [20]. A massa corporal e a estatura foram utilizadas no cálculo do IMC, sendo excluídos os indivíduos com IMC menor que 18,5 e maior que $24,9 \mathrm{~kg} / \mathrm{m}^{2}$.

- Questionário Internacional de Atividade Física (IPAQ): utilizado para avaliar o nível de atividade física, classificando os indivíduos em: sedentário, insuficiente ativo $A$ ou $B$, ativo e muito ativo [20]. Os ativos e/ou muito ativos foram excluídos do estudo.

- Espirometria: realizada pelo espirômetro portátil (One Flow FVC KIT, Clement Clark Internacional, Reino Unido). Os procedimentos técnicos, critérios de aceitabilidade e reprodutibilidade seguiram as diretrizes para testes de função pulmonar da Sociedade Brasileira de Pneumologia e Tisiologia [21] e interpretados segundo os valores referenciais para a população brasileira [15]. Os indivíduos foram instruídos a colocar o bocal entre os dentes, sobre a língua, lábios firmemente posicionados ao redor do bocal sem escape aéreo. Sustentando o equipamento na posição horizontal, deveriam realizar uma inspiração ao nível da CPT, posicionar o bocal e, imediatamente, realizar uma expiração o mais rápida possível, sustentada por aproximadamente seis segundos. A manobra foi acompanhada por estímulo verbal vigoroso por parte do examinador. Os indivíduos permaneceram na posição sentada, pés apoiados no solo, usando clipe nasal, sendo a manobra repetida no mínimo, três e, no máximo, oito vezes, com intervalo de um minuto de descanso entre as manobras [21]. Os parâmetros espirométricos investigados foram a CVF, VEF1, relação VEF1/ CVF, PFE e Fluxo Expiratório Forçado entre $25 \%-75 \%$ da CVF (FEF $25-75 \%)$.

- Manovacuometria: a Pressão Inspiratória Máxima (PImáx) e Pressão Expiratória Máxima ( $\left(\mathrm{E}_{\text {máx }}\right)$ foram avaliadas pelo manovacuômetro MVD300 (Globalmed®), Porto Alegre/RS, Brasil). Utilizou-se um bocal de plástico rígido (Globalmed®, Porto Alegre, $\mathrm{RS}$, Brasil) com orifício de $2 \mathrm{~mm}$ de diâmetro [18], conectado à peça de conexão ao ambiente (rescal). Com o bocal entre os dentes, clipe nasal [16,17] e orifício do rescal aberto, solicitou-se uma exalação até o Volume Residual (VR), momento em que o orifício do rescal foi ocluído e na sequência solicitada uma inalação máxima, sustentada por cerca de 2 segundos para a medida de PImáx. Para medir a PEmáx, solicitou-se uma inspiração até a CPT [18] com o orifício do rescal aberto, na sequência oclusão do rescal seguida de uma exalação máxima sustentada por cerca de 2 segundos [18,22]. Foram realizados de três a cinco esforços máximos, sob estímulo verbal vigoroso, com intervalo de um minuto de descanso. Destes, 
selecionaram-se três esforços aceitáveis e reprodutíveis (diferença ? 10\%), registrouse o maior valor absoluto de pico [16,18], e comparou-se aos valores previstos [16].

- Pico de fluxo inspiratório nasal: o PFIN (In-Check Inspiratory Flow Meter, Clement Clarke Internacional, Reino Unido) foi medido a partir de uma expiração completa (até o VR), seguida de uma inspiração nasal tão rápida e forte quanto possível, com a boca fechada e a máscara bem adaptada à face $[14,23]$. O teste foi repetido no mínimo três vezes ou até se obterem três medidas tecnicamente satisfatórias, com variação menor que $10 \%$ e registrado o maior valor. O intervalo entre as mensurações foi de 30 segundos [23].

- Eletromiografia: pela eletromiografia de superfície (sEMG) registrou-se a atividade dos músculos esternocleidomastoideo (ECM) e trapézio superior (TS). Os procedimentos de aquisição do sinal EMG seguiram as recomendações da Surface ElectroMyoGraphy for the Non-Invasive Assessment of Muscles [24].

O local da coleta foi equipado com piso emborrachado, lâmpadas e telefones celulares desligados e removidos quaisquer acessórios de metal dos partipantes. Os exames foram realizados na posição sentada em uma cadeira adaptada [7], tronco ereto, braços relaxados, olhos abertos orientados pelo plano de Frankfurt e pés apoiados no solo.

Previamente à fixação dos eletrodos, realizou-se a limpeza da pele com gaze embebida em álcool $70 \%$. Utilizaram-se sensores pré-amplificados com entrada diferencial ligados a eletrodos adesivos modelo Double $(\mathrm{Ag} / \mathrm{AgCl})$, bipolar, diâmetro de $10 \mathrm{~mm}$ e distância interpolos de $20 \mathrm{~mm}$ (Hall Indústria e Comércio). Um eletrodo de referência (Meditrace 100) foi posicionado no manúbrio esternal [24].

Para o ECM os eletrodos foram posicionados longitudinalmente às fibras, no ponto médio do ventre muscular e, para o TS, no ponto médio de uma linha traçada entre o processo espinhoso da sétima vértebra cervical e o acrômio [24].

Os sinais EMG foram obtidos pelo Miotool 400 (Miotec®, Porto Alegre, Brasil), sistema de quatro canais, 14 bites de resolução, frequência de amostragem de $2000 \mathrm{~Hz}$ por canal, filtro tipo Butterworth de 4 a ordem e passa-banda com frequência de corte de $20 \mathrm{~Hz}$ a $500 \mathrm{~Hz}$ [24]. O sinal foi registrado pelo Software Miograph (Miotec $\AA$, Porto Alegre, Brasil) e transmitido a um notebook (HP 420) Intel Celeron via porta USB. Os equipamentos foram ligados com bateria própria, sem conexão com a rede elétrica.

As coletas foram realizadas durante os testes de PFIN e $\mathrm{PI}_{\text {máx }}$, repetidos por pelo menos três vezes, com intervalo de dois minutos. Para a normalização do sinal, utilizou-se o registro da Contração Voluntária Máxima (CVM), obtido durante a flexão anterior da cabeça (ECM) e a elevação dos ombros (TS), contra uma resistência oferecida por anteparos existentes na cadeira adaptada [7]. Esta foi registrada durante cinco segundos e repetida por pelo menos duas vezes, com intervalo de dois minutos.

A atividade muscular foi analisada no domínio da amplitude, representada pela raiz quadrada da média (Root Mean Square (RMS)) do sinal EMG, expressa em microvolt e transformada em valores relativos de um valor de referência (CVM). Os dados foram processados por meio do Software MATLAB (The MathWorks® versão 7.6.0 R2008a).

Cálculo amostral: foi obtido a partir do estudo piloto com 10 indivíduos em cada grupo considerando os resultados da sEMG do ECM (maior coeficiente de variação), nível de significância de $5 \%$ e poder de $80 \%$ (software WinPepi, versão 1.5) e previu uma amostra com 22 indivíduos em cada grupo.

Análise estatística: os dados foram analisados pelos testes Shapiro-Wilk (normalidade), $\mathrm{t}$ student e Mann-Whitney (comparação entre os grupos) e de Spearman (correlação entre as variáveis). 
Amostra composta por 77 voluntários, 39 (28 mulheres) no grupo RN e 38 (25 mulheres) no grupo RO. Todos os integrantes do grupo RO foram classificados como RO funcionais, ou seja, de causa não orgânica, principalmente problemas alérgicos e hábitos parafuncionais. Pelo IPAQ todos foram classificados como insuficiente ativos.

As características antropométricas, de permeabilidade nasal e pressões respiratórias máximas apresentadas na Tabela I mostram que a permeabilidade nasal (PFIN) e a pressão

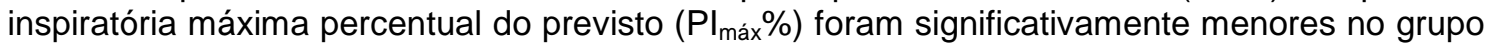
RO (Tabela I).

Tabela I - Características antropométricas, de permeabilidade nasal e de pressões respiratórias máximas.

\begin{tabular}{|c|c|c|c|}
\hline & $\begin{array}{l}\text { Grupo RN } \\
(n=39)\end{array}$ & $\begin{array}{l}\text { Grupo RO } \\
(\mathrm{n}=38)\end{array}$ & \\
\hline & Média_DP & Média土DP & p-valor \\
\hline Idade (anos) & $22,6 \pm 2,9$ & $22,7 \pm 3,5$ & 0,99 \\
\hline Massa Corporal (kg) & $59,9 \pm 11,1$ & $65 \pm 12,2$ & 0,05 \\
\hline Estatura (m) & $1,68 \pm 0,09$ & $1,69 \pm 0,1$ & 0,45 \\
\hline $\operatorname{IMC}\left(\mathrm{kg} / \mathrm{m}^{2}\right)$ & $21,1 \pm 2,3$ & $22,5 \pm 2,8$ & $0,03^{*}$ \\
\hline PFIN (I/min) & $147,7 \pm 35,6$ & $121,4 \pm 31$ & $<0,01^{* *}$ \\
\hline PImáx obtida $\left(\mathrm{cmH}_{2} \mathrm{O}\right)$ & $-109,82 \pm 20,53$ & $-101,79 \pm 22,07$ & 0,10 \\
\hline PImáx \% previsto & $100,2 \pm 14,5$ & $91 \pm 14,8$ & $<0,01^{*}$ \\
\hline PEmáx obtida $\left(\mathrm{cmH}_{2} \mathrm{O}\right)$ & $117,56 \pm 29,81$ & $106,50 \pm 25,82$ & 0,08 \\
\hline PEmáx \% previsto & $102,6 \pm 17,1$ & $91,6 \pm 18$ & $<0,01^{\star}$ \\
\hline
\end{tabular}

$\mathrm{RN}=$ respirador nasal; $\mathrm{RO}=$ respirador oral; $\mathrm{IMC}=$ Índice de massa corporal; PFIN = Pico de fluxo inspiratório nasal; PImáx = pressão inspiratória máxima; PEmáx = pressão expiratória máxima; *teste t-student; **Mann-Whitney.

Ambos os grupos apresentaram valores espirométricos nos parâmetros da normalidade, no entanto, verificou-se diferença significativa entre os grupos, com menor CVF\% e VEF1\% no grupo RO (Tabela II).

Tabela II - Volumes e capacidades pulmonares obtidos pela espirometria nos grupos $R N$ e $R O$.

\begin{tabular}{|c|c|c|c|}
\hline & $\begin{array}{l}\text { Grupo RN } \\
(n=39)\end{array}$ & $\begin{array}{l}\text { Grupo RO } \\
(n=38)\end{array}$ & \\
\hline & Média土DP & Média土DP & p-valor \\
\hline PFE obtido (I/s) & $485,26 \pm 110,48$ & $478,68 \pm 101,60$ & 0,81 \\
\hline PFE \% previsto & $102,59 \pm 12,08$ & $97,28 \pm 12,07$ & 0,05 \\
\hline VEF $_{1}$ obtido (I/s) & $3,60 \pm 0,71$ & $3,57 \pm 0,69$ & 0,89 \\
\hline VEF $_{1} \%$ previsto & $101,23 \pm 9,96$ & $96,84 \pm 8,00$ & $0,04^{*}$ \\
\hline CVF obtida (I) & $4,12 \pm 0,84$ & $4,05 \pm 0,84$ & 0,75 \\
\hline CVF \% previsto & $100,23 \pm 9,72$ & $94,59 \pm 8,64$ & $<0,01^{*}$ \\
\hline VEF $_{1} /$ CVF (\%) & $87,72 \pm 6,60$ & $88,44 \pm 6,71$ & 0,82 \\
\hline FEF $_{25-75}$ obtido (I/s) & $4,43 \pm 0,94$ & $4,62 \pm 1,06$ & 0,42 \\
\hline FEF \% previsto & $100,76 \pm 24,65$ & $102,97 \pm 20,56$ & 0,92 \\
\hline
\end{tabular}

$\mathrm{RN}=$ respirador nasal; $\mathrm{RO}$ = respirador oral; $\mathrm{DP}$ = desvio padrão; PFE = pico de fluxo expiratório; VEF1 = volume expiratório forçado no primeiro segundo; CVF = capacidade vital forçada; FEF = fluxo expiratório forçado entre $25-75 \%$ da CVF; $\%=$ percentual do previsto; $I / s=$ litros por segundos; I = litros; *teste $t$ de student; $d=d$ de Cohen, tamanho de efeito; $\mathrm{P}=$ poder estatístico.

A atividade eletromiográfica do músculo esternocleidomastoideo durante os testes de PFIN e $\mathrm{PI}_{\text {máx }}$ foi significativamente menor no grupo $\mathrm{RO}$ quando comparado ao grupo $\mathrm{RN}$ (Tabela III). 
Tabela III - Valores normalizados (\%) do registro EMG dos músculos acessórios da inspiração.

\begin{tabular}{lllll}
\hline Atividade & Músculos & Grupo RN $(\mathbf{n}=\mathbf{3 9})$ & Grupo RO $(\mathbf{n}=\mathbf{3 8})$ & \\
\cline { 3 - 5 } PFIN & & Média \pm DP & Média $\pm D P$ & -valor \\
& ECM D & $109,2 \pm 57,8$ & $75,2 \pm 46,3$ & $<0,01^{* *}$ \\
& ECM E & $101,1 \pm 46$ & $71,4 \pm 43,6$ & $<0,01^{* *}$ \\
\multirow{4}{*}{ PImáx } & TS D & $13,3 \pm 12,4$ & $13,4 \pm 12,3$ & 0,94 \\
& TS E & $11,3 \pm 9,3$ & $12 \pm 10,3$ & 0,71 \\
& ECM D & $98,2 \pm 46,8$ & $70,9 \pm 37,9$ & $<0,01^{*}$ \\
& ECM E & $93 \pm 49,2$ & $65,9 \pm 32,3$ & $<0,01^{*}$ \\
& TS D & $17,2 \pm 16,7$ & $21,1 \pm 18,3$ & 0,80 \\
& TS E & $15,2 \pm 13,2$ & $16,3 \pm 15,2$ & 0,85 \\
\hline
\end{tabular}

$\mathrm{RN}=$ respirador nasal; $\mathrm{RO}=$ respirador oral; PFIN = pico de fluxo inspiratório nasal; PImáx = pressão inspiratória máxima; $\mathrm{ECM}$ = esternocleidomastoideo; $\mathrm{TS}$ = trapézio superior; $\mathrm{D}=$ direito; $\mathrm{E}=$ esquerdo; ${ }^{*}$ teste $\mathrm{t}$-student; ${ }^{* *}$ teste de Mann-Whitney.

A correlação positiva entre PImáx e PFIN $(r=0,48 ; p<0,01)$ foi classificada como moderada (Figura 1).

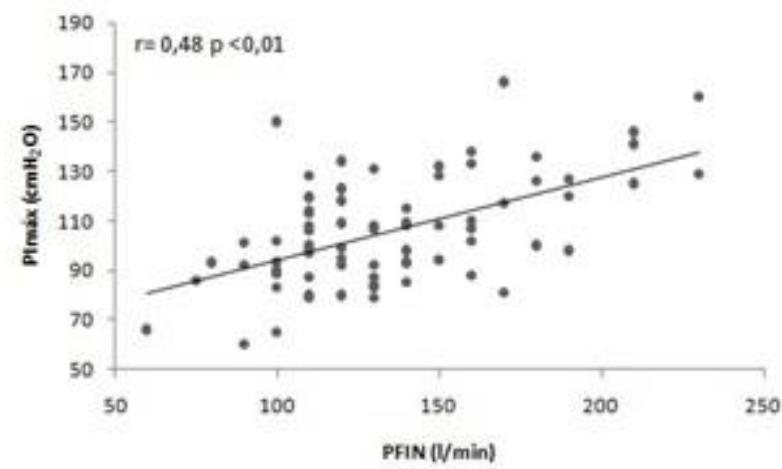

PImáx = pressão inspiratória máxima; PFIN = pico de fluxo inspiratório nasal

Figura 1 - Correlação entre PImáx e PFIN.

Discussão

Os respiradores orais ao serem comparados com os respiradores nasais apresentaram menores valores no PFIN, CVF\% e VEF1\%, $\mathrm{PI}_{\text {máx }}$ e $\mathrm{PE}_{\text {máx }}$ e menor ativação dos músculos acessórios da inspiração durante manobras respiratórias rápidas.

Em relação ao PFIN, observamos que os resultados são semelhantes aos evidenciados em estudo anterior [25], ao comparar adultos com e sem obstrução nasal por rinite. Demonstraram que o grupo com rinite obteve PFIN mais baixo (114 vs. $154 \mathrm{l} / \mathrm{min}$ ), assim como na comparação de pacientes com e sem queixas de obstrução nasal (123 vs $151 \mathrm{l} / \mathrm{min}$ ). Estudo [26] que comparou um grupo de pacientes com rinossinusite crônica antes e após cirurgia endoscópica nasal observou aumento significativo do PFIN (80,8 vs. $111,2 \mathrm{l} / \mathrm{min})$ mostrando que o procedimento cirúrgico foi efetivo. Outro estudo [27] avaliou os efeitos do dilatador nasal externo sobre o PFIN, resistência nasal e capacidade aeróbia em atletas saudáveis e com rinite alérgica. Os resultados mostraram que o dispositivo aumentou significativamente o PFIN, reduziu a resistência nasal e aumentou a capacidade de exercício tanto nos atletas saudáveis quanto nos com rinite.

A literatura mostra correlação inversa $(r=-0,34 ; p<0,01)$ do PFIN com os escores da escala de percepção de obstrução nasal (escala NOSE), evidenciando que à medida que diminui a patência nasal, pior é a percepção dos sintomas de obstrução [28]. Associação significativa entre o PFIN e sensação subjetiva de obstrução nasal (escala análogo-visual) mostra que à medida que aumenta a sensação de obstrução nasal diminuem os valores do PFIN [14]. Correlações entre métodos objetivos e subjetivos de avaliação da obstrução nasal também foram encontradas em outros estudos [29,30]. A avaliação do PFIN pode ser um método confiável para detectar e quantificar aumento da resistência nas vias aéreas superiores sendo tão importante quanto às mensurações objetivas da função pulmonar [31]. Ainda, 
permite o controle da homeostase e dos reflexos de congestão nasal, bem como dos efeitos do ambiente sobre a patência nasal.

Neste estudo observamos correlação positiva do PFIN com a $P I_{\text {máx }}(r=0,48 ; p<0,01)$ mostrando que redução da patência nasal (PFIN reduzido) está relacionada com redução da força muscular inspiratória $\left(\mathrm{PI}_{\text {máx }}\right.$ reduzida), demonstrando que avaliações objetivas $\mathrm{e}$ subjetivas agregam informações diferentes que se complementam e podem ser consideradas na prática clínica [32].

Uma limitação no uso do PFIN para medir a permeabilidade nasal é quando o indivíduo tem alteração na função respiratória com redução da capacidade pulmonar [23]. Por isso, a necessidade da espirometria complementar dessa avaliação para confirmar se 0 comprometimento respiratório é devido exclusivamente à obstrução das vias aéreas superiores.

Embora dentro dos valores de normalidade [15] as médias de VEF1\% e CVF\% foram mais baixas no grupo RO, esta diferença chama a atenção para a importância de estudos longitudinais que avaliem a função pulmonar de indivíduos com sintomatologia de obstrução nasal, ao longo do tempo, possibilitando a detecção de um declínio funcional que seria esperado mais tardiamente pelo envelhecimento cronológico. No estudo atual não houve correlação do PFIN com as variáveis espirométricas relacionadas com a permeabilidade das vias aéreas inferiores, mostrando que as mesmas não influenciaram na redução PFIN dos RO.

As médias das $\mathrm{PI}_{\text {máx }}$ e $P E_{\text {máx }}$ se apresentaram dentro dos valores preditos [16], porém, menores no grupo $\mathrm{RO}$. Tais resultados são similares aos de estudos com crianças $\mathrm{RO}[3,9]$ e com adultos $\mathrm{RO}$ [8], que apresentaram valores mais baixos na $\mathrm{PI}_{\text {máx }}(56 \% ; 74 \%)$ e $\mathrm{PE}_{\text {máx }}(66 \%$; 84\%) comparados com RN, sendo estes inferiores aos do presente estudo. Os autores [8] sugerem que a redução nas $\mathrm{PI}_{\text {máx }}$ e $\mathrm{PE}_{\text {máx }}$ tenha relação com a postura anteriorizada da cabeça e demais adaptações posturais decorrentes da respiração oral. As alterações posturais modificam o comprimento das fibras musculares, o padrão de contração diafragmático e dos abdominais, culminando com redução na força. Ainda, a menor resistência oferecida pela cavidade oral à passagem de ar, reflete em menor esforço muscular e, consequentemente, redução da $\mathrm{PI}_{\text {máx }}$ e $P E_{\text {máx }}[9]$.

O sinal EMG mostrou menor ativação dos músculos inspiratórios acessórios no grupo $\mathrm{RO}$, durante os testes de inspiração rápida. Estudos prévios [33,34] com indivíduos saudáveis mostraram aumento da atividade EMG desses músculos, especialmente do ECM, com o aumento da velocidade do fluxo e consequente aumento da carga de trabalho respiratório. Assim, inspirações rápidas e bruscas requerem uma ativação mais intensa dos músculos ECM [33] que respondem com ativação de maior número de unidades motoras (UM) [35].

A literatura mostra que exercícios de curta duração e alta intensidade envolvem principalmente a ativação de UM do tipo II, predominantes nos músculos inspiratórios acessórios $[35,36]$. No presente estudo, os $\mathrm{RO}$ não apresentaram o mesmo padrão de ativação EMG observada em indivíduos saudáveis, uma vez que apresentaram menor recrutamento muscular que os RN. Isso pode ser devido ao fato de que além da carga de trabalho decorrente da maior resistência nasal, somou-se a carga imposta pelo aumento da velocidade do fluxo nos testes de inspiração rápida, resultando em uma carga excessivamente alta a ser vencida por músculos previamente mais fracos que os do grupo RN. Outra justificativa para os resultados, embora não tenha sido objeto de investigação deste estudo, é a influência da RO sobre a postura craniocervical e a desvantagem mecânica à produção de força pela alteração na relação comprimento-tensão do músculo [37].

O presente estudo traz como contribuição para a prática clínica a importância de uma investigação global do modo respiratório, não envolvendo apenas intervenções médicas, ortodônticas e miofuncionais, mas também os aspectos ventilatórios e musculares, sobre os quais se identificou a influência da RO. Atualmente, a avaliação, bem como o tratamento dessa condição, ainda é abordada de forma fragmentada, restringindo-se a aspectos específicos de cada profissional envolvido. Por se tratar de uma condição frequente na infância e que tende a se manter na idade adulta, evidencia-se a necessidade da conscientização dos pais, professores, pacientes e profissionais sobre a repercussão sistêmica da mesma e a possível interferência na qualidade de vida.

A hipótese de que existe diferença conforme o modo ventilatório, ou seja, respiradores orais apresentem menores valores no PFIN, nas pressões respiratórias máximas e nas variáveis espirométricas, foi confirmada. No entanto, não se confirmou a hipótese de que haja maior recrutamento da musculatura acessória da respiração, em relação aos respiradores nasais, resultado que é contraditório ao encontrado na literatura, especialmente com crianças respiradoras orais. 
Os adultos com respiração oral apresentaram menor pico de fluxo inspiratório nasal de pressões respiratórias máximas e de atividade elétrica dos músculos inspiratórios acessórios, quando comparados com os respiradores nasais. Verificou-se ainda que à medida que diminui o pico de fluxo inspiratório nasal, diminuem também as pressões respiratórias.

1. Cuccia AM, Lotti M, Caradonna D. Oral breathing and head posture. Angle Orthod 2008;78(1):77-82.

2. Berwig LC, Silva AMT. Análise quantitativa do palato duro em respiradores orais: revisão de literatura. Rev Soc Bras Fonoaudiol 2011;16(4):483-7.

3. Okuro RT, Morcillo AM, Sakano E, Schivinski CIS, Ribeiro MAGO, Ribeiro JD. Exercise capacity, respiratory mechanics and posture in mouth breathers. Braz J Otorhinolaryngol 2011;77(5):656-62.

4. Yi LC, Jardim JR, Inoue DP, Pignatari SS. The relationship between excursion of the diaphragm and curvatures of the spinal column in mouth breathing children. $\mathrm{J}$ Pediatr 2008;84(2):171-7.

5. Pires MG, Di Francesco RC, Junior Mello JF, Grumach AS. Alterações torácicas secundárias ao aumento de volume de tonsilas palatinas e faríngeas. Arq Int Otorhinolaryngol 2007;11(2):99-105.

6. Ribeiro EC, Marchiori SC, Silva AM. Electromyographic analysis of trapezius and sternocleidomastoideus muscles during nasal and oral inspiration in nasal- and mouthbreathing children. J Electromyogr Kinesiol 2002;12(4):305-16.

7. Corrêa EC, Bérzin F. Mouth breathing syndrome: cervical muscles recruitment during nasal inspiration before and after respiratory and postural exercises on swiss ball. Int $J$ Pediatr Otorhinolaryngol 2008;72(9):1335-43.

8. Milanesi JM, Weber P, Berwig LC, Ritzel RA, Silva AMT, Corrêa ECR. Childhood mouth-breathing consequences at adult age: ventilatory function and quality of life. Fisioter Mov 2014;27(2):211-8.

9. Pires MG, Di Francesco RC, Grumach AS, Mello JF Jr. Evaluation of inspiratory pressure in children with enlarged tonsils and adenoids. Braz J Otorhinolaryngol 2005;71(5):598-601.

10. Barbiero EF, Vanderlei LCM, Nascimento PC, Costa MM, Scalabrini Neto A. Influência do biofeedback respiratório associado ao padrão quiet breathing sobre a função pulmonar e hábitos de respiradores bucais funcionais. Rev Bras Fisioter 2007;11(5):347-53.

11. Silveira W, Mello FCQ, Guimarães FS, de Menezes SLS. Postural alterations and pulmonary function of mouth-breathing children. Braz J Otorhinolaryngol 2010;76(6):683-6.

12. Braun T, Rich M, Kramer MF. Correlation of three variables describing nasal patency (HD, MCA, NOSE score) in healthy subjects. Braz J Otorhinolaryngol 2013;79(3):354-8.

13. Mendes AI, Wandalsen GF,Solé D. Objective and subjective assessments of nasal obstruction in children and adolescents with allergic rhinitis. J Pediatr 2012;88(5):38995.

14. Kjærgaard $T$, Cvancarova M, Steinsvåg SK. Does nasal obstruction mean that the nose is obstructed? The Laryngoscope 2008;118(8):1476-81.

15. Pereira, CAC. Espirometria. J Bras Pneumol 2002;28(3):S1-S82.

16. Neder JA, Andreoni S, Lerario MC, Nery LE. Reference values for lung function tests, II: maximal respiratory pressures and voluntary ventilation. Braz J Med Biol Res 1999;32:719-27.

17. Parreira VF, França DC, Zampa CC, Fonseca MM, Tomich GM, Britto RR. Pressões respiratórias máximas: valores encontrados e preditos em indivíduos saudáveis. Rev Bras Fisioter 2007;11(5):36-8.

18. Souza RB. Pressões respiratórias estáticas máximas. J Bras Pneumol 2002;28(3):S155-65.

19. Ribeiro EC, Marchiori SC, Silva AM. Electromyographic muscle EMG activity in mouth and nasal breathing children. Cranio 2004;22(2):145-50. 
20. Matsudo S, Araújo T, Matsudo V, Andrade D, Andrade E, Oliveira LC, et al. International physical activity questionnaire (IPAQ): study of validity and reability in Brazil. Rev Bras Ativ Fis Saúde 2001;6(2):5-18.

21. Pereira CAC, Neder JA. Diretrizes para testes de função pulmonar. J Bras Pneumol 2002;28(3):S1-S238.

22. Coelho CM, Carvalho RM, Gouvêa DSA, Marques JNJ. Comparação entre parâmetros de pressões respiratórias máximas em indivíduos saudáveis. J Bras Pneumol 2012;38(5):605-13.

23. Ottaviano G, Lund VJ, Coles S, Staffieri A, Scadding G. Does peak nasal inspiratory flow relate to peak expiratory flow? Rhinology 2008;46(3):200-3.

24. Hermens JH, Freriks B, Disselhorst-Klug C, Rau G. Development of recommendations for SEMG sensors and sensor placement procedures. J Electromyogr Kinesiol 2000;10(5):361-74.

25. Teixeira RUF, Zappelini CE, Alves FS, Costa EA. Peak Nasal Inspiratory Flow Evaluation as an objective method of measuring nasal airflow. Braz $\mathrm{J}$ Otorhinolaryngol $2011 ; 77(4): 473-80$.

26. Whitcroft KL, Andrews PJ, Randhawa OS. Peak nasal inspiratory flow correlates with quality of live in functional endoscopic sinus surgery. Clin Otolaryngol 2017 [epub ahead of print].

27. Dinardi RR, de Andrade CR, Ibiapina CC. Effect of the external nasal dilator on adolescent athletes with and without allergic rhinitis. Int J Pediatr Otorhinolaryngol 2017;97:127-34.

28. Trevisan ME, Bellinaso JH, Pacheco AB, Augé LB, Silva AMT, Corrêa ECR. Respiratory mode, nasal patency and palatine dimensions. CoDas 2015;27(2):201-6.

29. Teixeira RU, Zappelini CE, Alves FS, da Costa EA. Peak Nasal Inspiratory Flow Evaluation as an objective method of measuring nasal airflow. Braz $\mathrm{J}$ Otorhinolaryngol 2011;77(4):473-80.

30. Kahveci OK, Miman MC, Yucel A, Yucedag F, Okur E, Altuntas A. The efficiency of nose obstruction symptom evaluation (NOSE) scale on patients with nasal septal deviation. Auris Nasus Larynx 2012;39(3):275-9.

31. Ottaviano G, Fokkens WJ. Measurements of nasal airflow and patency: a critical

32. review with emphasis on the use of peak nasal inspiratory flow in daily practice.

33. Allergy 2016;71(2):162-74.

34. Braun T, Rich M, Kramer MF. Correlation of three variables describing nasal patency (HD, MCA, NOSE score) in healthy subjects. Braz J Otorhinolaryngol 2013;79(3):354-8.

35. Costa D, Vitti M, Tossello DO, Costa RP. Participation of the sternocleidomastoid muscle on deep inspiration in man. An electromyographic study. Rev Bras Fisioter 1997;2(1):43-7.

36. Tomich GM, França DC, Diório ACM, Britto RR,. Sampaio RF, Parreira VF. Breathing pattern, thoracoabdominal motion and muscular activity during three breathing exercises. Braz J Med Biol Res 2007;40:1409-17.

37. He ZH, Bottinelli R, Pellegrino MA, Ferenczi MA, Reggiani C. ATP consumption and efficiency of human single muscle fibers with different myosin isoform composition. Biophys J 2000;79:945-61.

38. Polla B, D'Antona G, Bottinelli R, Reggiani C. Respiratory muscle fibres: specialisation and plasticity. Thorax 2004;59(9):808-17.

39. Correia PP. Aparelho locomotor: função neuromuscular e adaptações à atividade física. Portugal: Edições FMH; 2012. 